\title{
Monitoring the inputs required to extend and sustain hygiene promotion: findings from the GLAAS 20I3/20 I 4 survey $\dagger$
}

\author{
Leslie D. Moreland ${ }^{1,2}$, Fiona M. Gore ${ }^{2}$, Nathalie Andre ${ }^{2}$, Sandy Cairncross' and Jeroen H. J. Ensink ${ }^{1, \dagger}$ \\ 1 Faculty of Infectious and Tropical Diseases, London School of Hygiene and Tropical Medicine, London, UK \\ 2 Water, Sanitation, Hygiene and Health Unit, World Health Organization, Geneva, Switzerland
}

\begin{abstract}
овJECTIVES There are significant gaps in information about the inputs required to effectively extend and sustain hygiene promotion activities to improve people's health outcomes through water, sanitation and hygiene (WASH) interventions. We sought to analyse current country and global trends in the use of key inputs required for effective and sustainable implementation of hygiene promotion to help guide hygiene promotion policy and decision-making after 2015 .

METHODS Data collected in response to the GLAAS 2013/2014 survey from 93 countries of 94 were included, and responses were analysed for 12 questions assessing the inputs and enabling environment for hygiene promotion under four thematic areas. Data were included and analysed from 20 External Support Agencies (ESA) of 23 collected through self-administered surveys. RESULTS Firstly, the data showed a large variation in the way in which hygiene promotion is defined and what constitutes key activities in this area. Secondly, challenges to implement hygiene promotion are considerable: include poor implementation of policies and plans, weak coordination mechanisms, human resource limitations and a lack of available hygiene promotion budget data.

CONCLUSION Despite the proven benefits of hand washing with soap, a critical hygiene-related factor in minimising infection, GLAAS 2013/2014 survey data showed that hygiene promotion remains a neglected component of WASH. Additional research to identify the context-specific strategies and inputs required to enhance the effectiveness of hygiene promotion at scale are needed. Improved data collection methods are also necessary to advance the availability and reliability of hygiene-specific information.
\end{abstract}

keywords GLAAS report, hygiene promotion, monitoring, financing, UN-Water, hygiene policy, human resources, governance

\section{Introduction}

Even though hygiene promotion is a critical component of almost any public health intervention, and has proven to be one of the most cost-effective health interventions, it remains a neglected issue [1]. Recent studies estimated that globally 842000 deaths could be attributed to a combination of water-, sanitation- and hygiene-related risk factors and that an estimated 297000 diarrhoearelated deaths were caused by inadequate hand hygiene [2, 3]. The Millennium Development Goal (MDG), target $7 \mathrm{c}$, has provided a platform for global monitoring efforts for access to water and sanitation, but no target for hygiene promotion was set [4]. As a result, the status of

$\dagger$ This paper is dedicated to Jeroen Ensink, for his passion and commitment working to improve WASH for all, and for his practical guidance and friendship. hygiene promotion and related activities have suffered and limited access to quality hygiene-specific data.

Recognising the need to enhance global monitoring efforts through better consolidation of information about water, sanitation and hygiene (WASH) inputs; combined data about trends from countries and external support agencies; and complementary output level data to that collected by the Joint Monitoring Programme (JMP) resulted in the first publication of the pilot UN-Water Global Analysis and Assessment for Sanitation and Drinking-Water (GLAAS) report in 2008 [5, 6]. Building on its first biannual publication, the GLAAS report has since highlighted the need for more investment in hygiene promotion and credible data [5-12]. As the MDGs come to an end in 2015, new WASH-specific targets have been proposed as part of the Sustainable Development Goals (SDGs), and this time they are likely to include hygienespecific targets [13]. The Demographic Health Surveys 
(DHS) and Multi-Indicator Cluster Surveys (MICS) have also recently started to collect information about people's hygiene practices, while hygiene promotion questions are now included in the GLAAS surveys, providing data on the global trends in hygiene promotion inputs by responding countries. Yet, if policy makers and other key stakeholders are to make informed decisions about how best to implement hygiene promotion initiatives in order to improve and save lives, a much better understanding of what national, regional and global hygiene inputs exist is required to drive progress in the WASH and health sectors.

Thus, while the report based on the hygiene questions in the 2011/2012 survey [7] focused on the shortcomings in the data and the lack of a serious approach to hygiene in many countries, our goal in this study was to understand the constraints and opportunities for promoting hygiene more effective and more widely applied. To improve our understanding of the status of hygiene promotion, we used data from the GLAAS 2013/2014 survey to determine what inputs were in place to support delivery of hygiene promotion programmes; to identify the factors contributing to the success, or failure, in their development and implementation; and to identify limitations in how hygiene promotion input-level data is collected and used, with specific reference to the GLAAS 2013/2014 survey.

\section{Methods}

As of October 2014, the GLAAS 2013/2014 survey was implemented in 94 countries through the support and facilitation of WHO at headquarters, regional and country level and in closer collaboration with partners (e.g. Water and Sanitation for Africa, WaterAid, UNICEF). For this research, 93 countries had responded as of June 23,2014 , representing a $26 \%$ increase in respondents from 2012. Respondents were from a variety of Ministries, and in most cases, for each country, one person self-identified as the primary contact for each of the relevant drinking water, sanitation and hygiene thematic areas who was in charge of coordinating responses for that particular section. A GLAAS national focal person was also assigned to coordinate country-level responses and submission. Qualitative and quantitative data were collected through multiple choice, direct and open-ended questions. The survey consisted of a total of 35 questions as part of four thematic areas: governance (13), monitoring (8), human resources (3) and finance (11). All responses were reviewed for internal consistency and completeness. Approximately ten participating countries were selected for a more thorough validation exercise to review GLAAS responses with available country documents.

Fourteen main questions assessing the inputs and enabling environment for hygiene promotion were analysed as part of the four thematic areas (Table 1 for a detailed list of the hygiene-specific survey questions that informed this study). Descriptive analysis was performed using Excel and Stata 13 (StataCorp, College Station, TX, USA). Summary results were tabulated by country and stratified by region; the MDG regions were used to do this stratification. ${ }^{1}$ For five questions, thematic coding was made using Excel; these included questions referencing policy documents, defining key terms and identifying sector priorities. Response rates for each question differed in completeness; thus, the number of countries responding to each question was referenced. $^{2}$

\section{Results}

\section{Governance}

Defining hygiene promotion. Of the 91 countries that responded, 80 reported on the type of hygiene promotion activities that are considered to be within the national target coverage, defined as the percentage of the population reached through hygiene promotion. There were a diverse number of activities included in the definition of what constitutes hygiene promotion, most commonly training and education activities targeted at households and communities, schools and health facilities $(76 \%)$. They comprised formation of family, community and/or school health groups, training of trainers and teaching how to prevent illness through key messages (Figure 1). A total of $48 \%$ of respondents reported inclusion of water- and sanitation-specific activities in hygiene promotion targets, while $36 \%$ mentioned hygiene-specific promotion activities. The data did not allow for stratification between individual, school or healthcare facility hygiene promotion activities. It only provided an overall summary of the key activities countries undertook as part of hygiene promotion, and did not allow for a more indepth analysis to compare activities between, or within, counties. Figure 2 illustrates the variety of hygiene promotion activities.

\footnotetext{
${ }^{1}$ Caucasus, Developed, Eastern Asia, LAC, Northern Africa, Oceania, South-eastern Asia, Southern Asia, Sub-Saharan Africa and Western Africa.

${ }^{2}$ For a full methodology refer to the GLAAS 2014 report, Annex A.
} 


\section{D. Moreland et al. Monitoring sustained hygiene promotion}

Table I Hygiene-specific questions in GLAAS survey 2013/2014

No.

Section A: Governance

1 A2: Do national policies and plans exist, and to what extent are these implemented to ensure the provision of water and sanitation?

A2 Hygiene promotion

A2 Hygiene promotion in schools

A2 Hygiene promotion in health facilities

A2 Other comments

2 A3: Please indicate the coverage target (including the year targets are expected to be attained) as documented in the policy or plan

A3 Target coverage hygiene promotion

A3 Policy name hygiene promotion

A3 Policy date hygiene promotion

A3 Target date hygiene promotion

A3 Target coverage hygiene promotion in schools

A3 Policy name hygiene promotion in schools

A3 Policy date hygiene promotion in schools

A3 Target date hygiene promotion in schools

A3 Target coverage hygiene promotion in health care facilities

A3 Policy name hygiene promotion in health care facilities

A3 Policy date hygiene promotion in health care facilities

A3 Target date hygiene promotion in health care facilities

A3 Other comments

3 A5: Please indicate what types of hygiene promotion activities are considered in your target coverage.

4 A9: Please list ministries/ national institutions with responsibilities in WASH and indicate the level of responsibility

in each sector (drinking-water, sanitation and hygiene). Please add lines or attach separate page if necessary.

$5 \quad$ A11: To what extent do NGOs coordinate with government institutions?

A11 Number of NGOs implementing WASH projects - hygiene

A11 Number of NGOs participating in central government-led sector coordination framework - hygiene

A11 Number of NGOs actively coordinating work with local authorities in support of government-defined priorities - hygiene

A11 Number of NGOs reporting results of monitoring back to government institutions - hygiene

A11 Additional notes

6 A12: Are there clearly defined procedures in laws or policies for participation by service users (e.g. households) and communities in planning programs and what is the level of participation?

A12 Procedures defined in law or policy - hygiene promotion

A12 Extent to which service users participate in planning - hygiene promotion

A12 Additional notes

Section B: Monitoring

7 B1: When was the last national sanitation and drinking-water supply review or assessment conducted (month/year)?

B1 No national assessment - hygiene promotion and/or practice

B1 Assessment date (month/year) - hygiene promotion and/or practice

B1 Please attach a copy of the applicable report (or provide web-link) - hygiene promotion and/or practice

$8 \quad$ B6 Are there clearly defined national standards or agreed upon performance indicators that are used in the following categories?

B6iii Performance indicators exist - hygiene promotion coverage

B6iii Performance indicator status - hygiene promotion coverage

B6iii ): Performance indicators exist - cost effectiveness of programs (e.g. costs, hygiene knowledge, hygiene practices

B6iii Performance indicator status - cost effectiveness of programs

B6iii Additional notes

Section C: Human Resources

$9 \quad$ C1 a) HR strategy existence: Does an overall HR strategy exist to develop and manage human resource in sanitation and drinking water?

C1 Hygiene - urban, rural

10 C3 What are the three (3) tasks that would most benefit from increased human resource capacity [for hygiene]?

C3 Policy development, institutional coordination, national and local/provincial WASH planning, construction

of facilities, operation and maintenance $(\mathrm{O} \& \mathrm{M})$, community mobilisation, financial planning and expenditure, enforcement of regulations, health promotion, monitoring and evaluation, other 


\section{D. Moreland et al. Monitoring sustained hygiene promotion}

Table I (Continued)

No.

Section D: Finance

11 D1: Has the government defined a financing plan/budget for the WASH sector, clearly assessing the available sources

of finance and strategies for financing future needs (i.e. who should pay for what), that is published and agreed?

D1 Hygiene promotion

D1 Other comments

12 D2 Please list ministries/ national institutions with responsibilities in WASH and budget.

13 D3: Are expenditures reports available that allow actual spending on WASH to be compared with committed funding - hygiene?

D3 Expenditure reports not available

D3 Expenditure reports available and include actual vs. committed for - ODA

D3 Expenditure reports available and include actual vs. committed for non ODA

D3 Expenditure reports available and include actual vs. committed for government expenditure

D3 If disaggregated reports on actual spending are not available please describe what is available

14 D9: Is there a coordination mechanism between bi-lateral/multi-lateral donors and government and how are the donor funds

channelled to the sector?

D9 Total number of NGOs involved - hygiene

D9 Number of NGOs allocating funding through a signed agreement responsive to government defined priorities

D9 Funding channels - number of donors using the following channels to fund the country WASH sector:

D9 Direct funding to sector not through national budget

D9 Targeted budget support for the sector (basket funding)

D9 General budget support with specific objectives or performance indication for the sector

D9 Please provide information on difficulties encountered in the coordination and channeling of the external funding

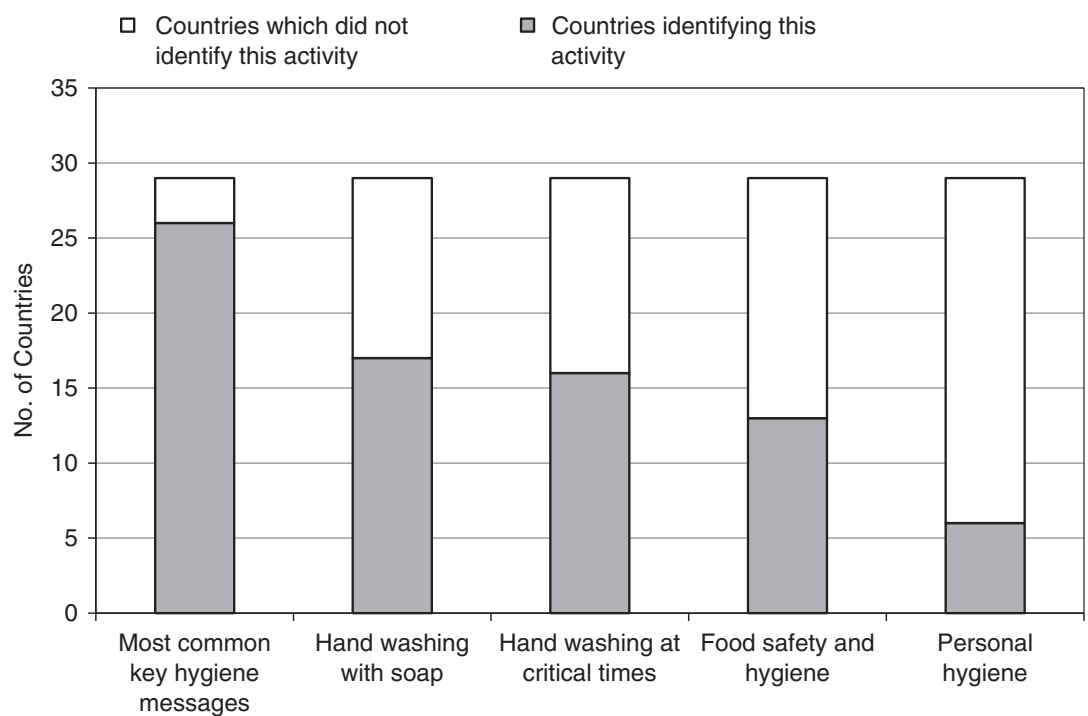

Figure I Most common key messages as part of hygiene-specific promotion activities.
Policy development, implementation and tar-

gets. National hygiene promotion policies were found in place in 71 of 86 countries $(83 \%)$, yet only $21 \%$ of countries had fully implemented these policies through the commitment of funding and regular review. Similar trends were found for plans and strategies for both school and healthcare facilities (Figure $3 b, c)$. A lack of funding was cited as a contributing factor to the lack of full implementation. Although $>80 \%$ of countries stated they had plans and policies in place, only $52 \%$ provided the name of the actual policy, or plan for hygiene promotion targets. This was even less common for school- and healthcare facility-specific policies. Figure 3 a shows where countries are in regard to implementation of hygiene promotion policies. 
Figure 2 Types of hygiene promotion activities considered in target coverage. Countries listed multiple indicators; thus, the total number is higher than the total number of respondents $(n=80)$. Other included mention of policy/standards, hygiene household surveys and vector/pest control.

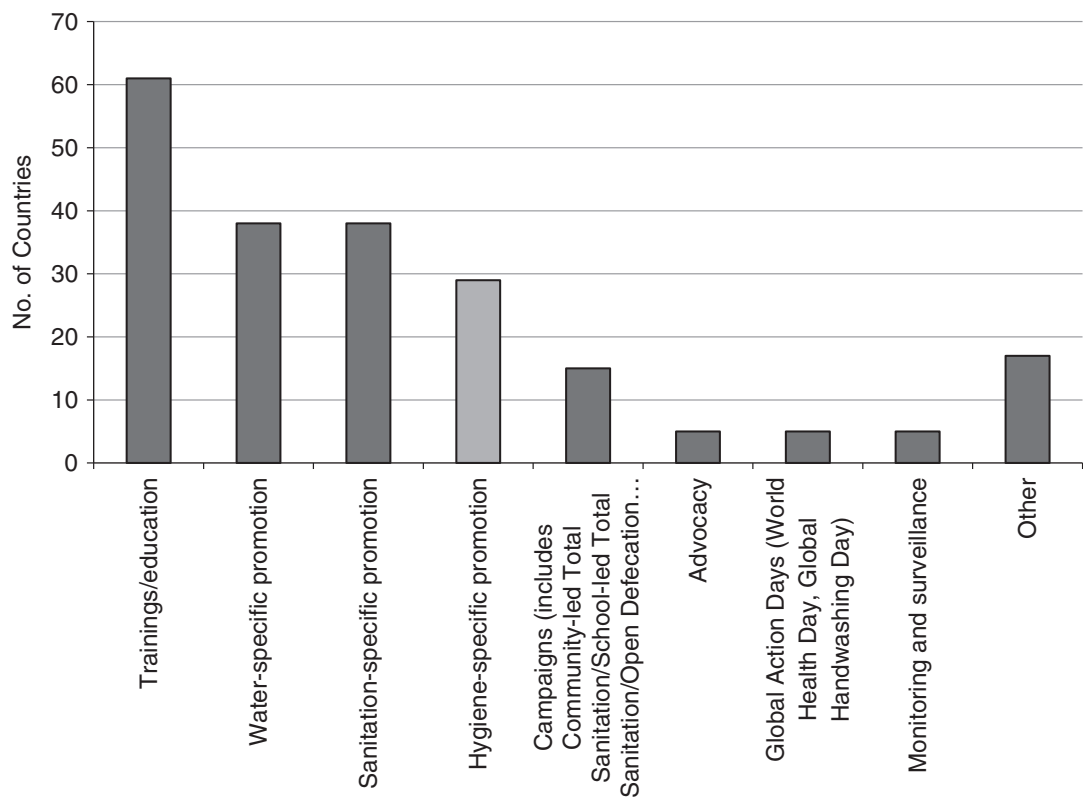

Of 91 countries, $50(55 \%)$ provided additional details about existing types of health and/or hygiene policies. Of these, 20 countries (36\%) specifically mentioned having a hygiene, or hygiene promotion policy, and 11 (20\%) countries reported having school-specific hygiene policies. Fewer than 10 countries each reported having hygiene as part of a general WASH policy (8), sanitation and health policy (7) and health facility policy (4).

Institutional coordination. Respondents indicated that hygiene promotion was both led and supported by many different ministries, ranging from Ministries of Health, Education and Rural Development to Ministries of Public Works and Housing, Water and Social Affairs. In several cases, countries indicated that two or more ministries had key leadership roles for hygiene promotion activities. Data also showed that programmes are managed at a federal, municipal and local level leading to a complex matrix of responsibility within countries to coordinate policies, budgets and activities.

Just under half the countries reported that non-governmental organizations (NGOs) participated in a central government-led sector coordination framework, and even fewer reported monitoring and evaluation results to government institutions. On average, 54\% participated in central government-led sector coordination frameworks, $55 \%$ actively coordinated work with local authorities in support of government-defined priorities and $45 \%$ reported results of monitoring and evaluation back to government institutions.
Community and user participation. The data indicated that while policies and procedures for participation in hygiene promotion by service users are in place in a high proportion of countries $(74 \%)$, $82 \%$ reported low-tomedium level of participation in hygiene promotion planning processes. Figure 4 shows the overall distribution of ways in which countries ranked the level of participation in these planning processes.

\section{Monitoring}

Performance indicators and tracking progress. The majority of countries responded that they were in the process of developing, or have agreed to apply, indicators to monitor both coverage and cost-effectiveness of hygiene promotion programmes. However, fewer than one-third of these countries track indicators against an established baseline for coverage of hygiene promotion, and only $5 \%$ track these indicators against an established baseline for cost-effectiveness of programmes. Table 2 shows the status of performance indicators used by respondent countries and highlights the difference in countries developing indicators and countries tracking indicators against an established baseline for monitoring cost-effectiveness of hygiene promotion programme. More than half of respondent countries reported national targets for hygiene promotion, almost double the number of countries $(26 \%)$ that reported using national targets to drive hygiene promotion programmes in 2012 [9]. 
L. D. Moreland et al. Monitoring sustained hygiene promotion
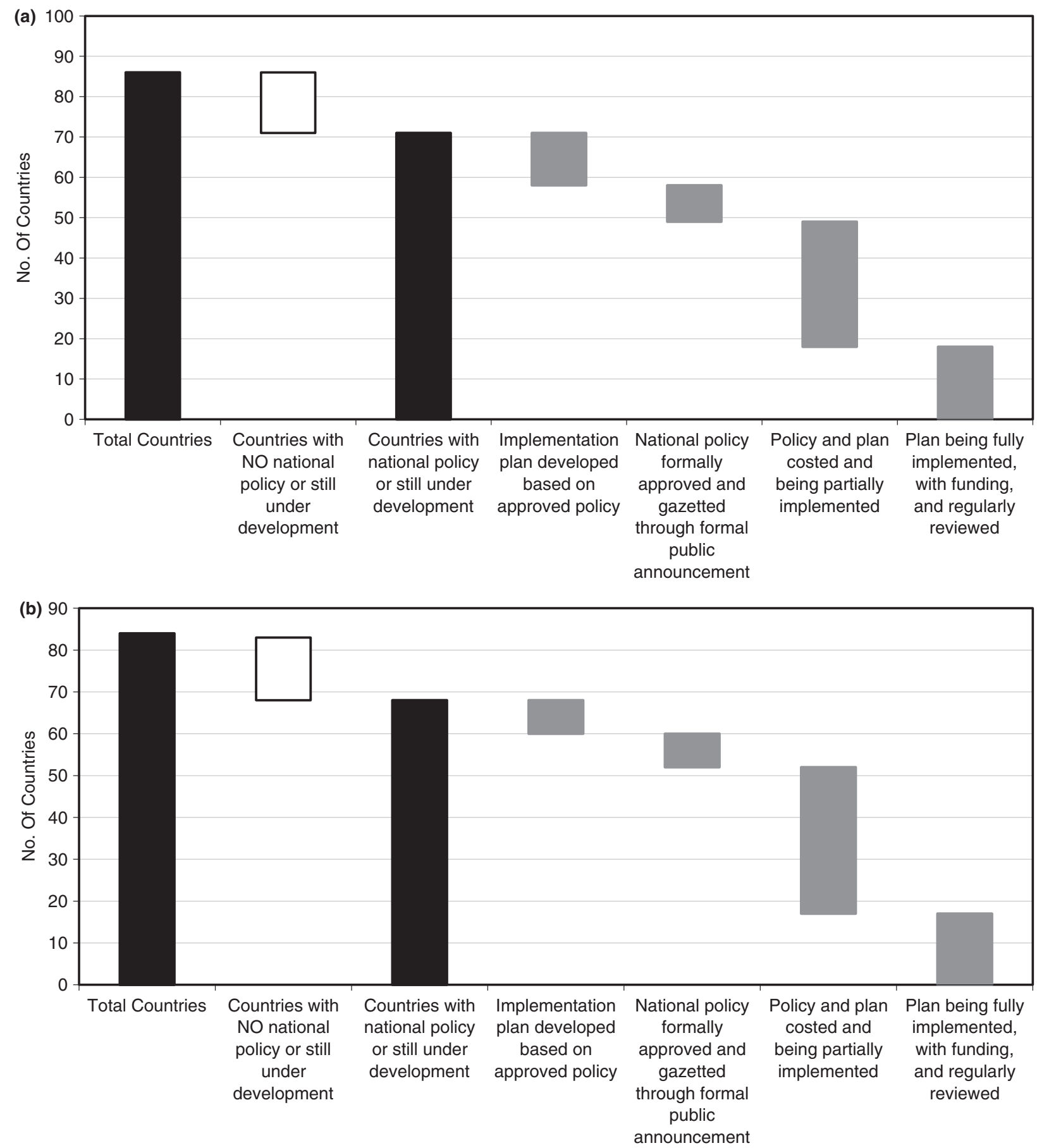

Figure 3 (a) Existence of national hygiene promotion policies and extent to which they are implemented $(n=86)$. (b) Existence of national school hygiene promotion policies and extent to which they are implemented $(n=84)$. (c) Existence of national healthcare facility hygiene promotion policies and extent to which they are implemented $(n=84)$. 
L. D. Moreland et al. Monitoring sustained hygiene promotion

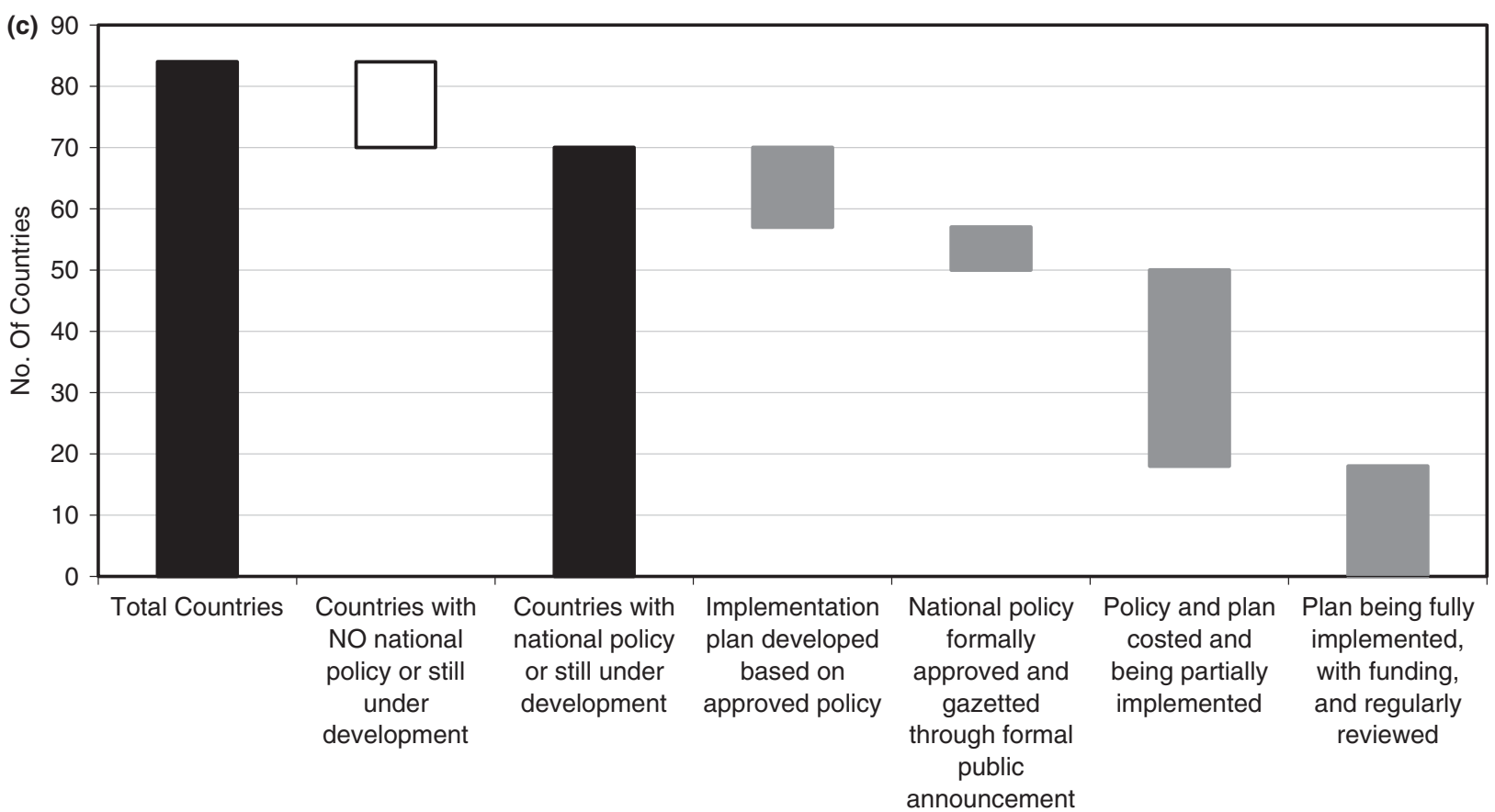

Figure 3 Continued.

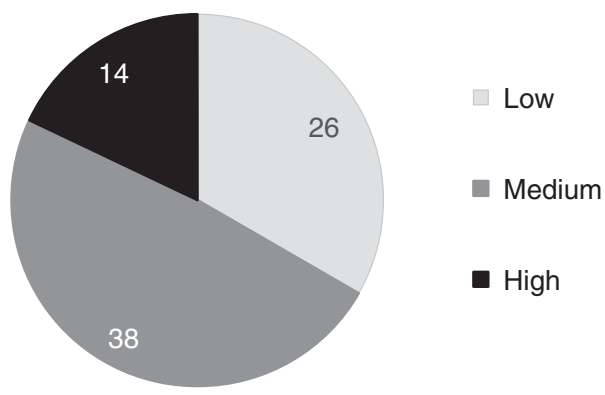

Figure 4 No. of countries and extent to which service users participate in planning $(n=78)$.

\section{Human resources}

Two-thirds of countries have not developed human resource (HR) strategies that cover all three WASH subsectors (sanitation, drinking water and hygiene). In addition to the challenge of a lack of policy or strategy, leading constraints to ensuring adequate WASH HR were as follows: inadequate remuneration, unwillingness to work in remote rural areas and the lack of skilled graduates. Figure 5 highlights the percentage of countries identifying tasks needing additional human resources; health promotion is the top concern under hygiene.

\section{Finance}

Existence of financing plan/budget. Although two-thirds of countries reported having a financing plan and/or budget for hygiene promotion, only $30 \%$ of countries adhered to, and consistently followed these. Only 10 of 53 respondent countries provided hygiene promotion budgets; many countries stated that it was difficult to disaggregate hygiene promotion budget lines from a combined WASH budget.

There was very little information available on comparing actual spending on WASH with committed funding. Thus, while several countries reported having specific budgets for hygiene promotion, and a small percentage of donors/external support agencies also reported investing in hygiene promotion as a priority area, there was a disconnect between tracking and reporting on financial flows specifically in this area.

External support agency/Donor funding. Of the total WASH aid commitments made by donors in 2012, only $1 \%$ was invested in hygiene education [11]. ${ }^{3}$ Aid

\footnotetext{
${ }^{3}$ Based on the data reported to the OECD using the purpose code 'education and training' where hygiene promotion around WASH is a subelement. There is a separate code in the OECD coding scheme for health education which can include elements of hygiene; in 2012, this amounted to USD 97 million.
} 


\section{D. Moreland et al. Monitoring sustained hygiene promotion}

Table 2 Defined national standards with status of performance indicators

\begin{tabular}{llllll}
\hline & & \multicolumn{2}{l}{ Of the countries monitoring hygiene promotion, performance indicators are as follows: } \\
\cline { 3 - 6 } & $\begin{array}{l}\text { No. of } \\
\text { respondents }\end{array}$ & $\begin{array}{l}\text { Being } \\
\text { developed }\end{array}$ & $\begin{array}{l}\text { Agreed but not } \\
\text { yet implemented }\end{array}$ & $\begin{array}{l}\text { Agreed and baseline } \\
\text { data established }\end{array}$ & $\begin{array}{l}\text { Agreed and tracked } \\
\text { against established baseline data }\end{array}$ \\
\hline $\begin{array}{l}\text { Coverage of hygiene } \\
\text { promotion programmes } \\
\begin{array}{c}\text { Cost effectiveness of hygiene } \\
\text { promotion programmes }\end{array}\end{array}$ & 71 & 19 & 2 & 14 & 17 \\
\hline
\end{tabular}

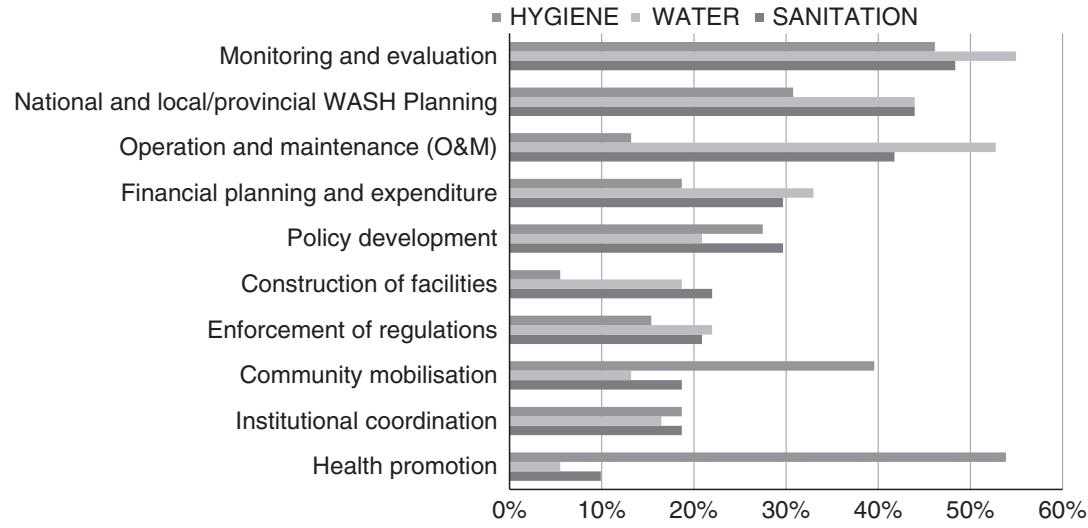

Figure 5 Percentage of countries identifying tasks needing additional human resources $(n=92)$. commitments for hygiene education were USD 38.9 million, vs. USD 3.8 billion for water and sanitation combined [11]. Of the 20 ESA respondents identifying the relative priority of drinking water, sanitation and hygiene promotion as part of the WASH portfolio, only $10 \%$ prioritized hygiene promotion. However, of the limited number of ESAs used in this analysis, a minority has started to respond to this critical gap. Countries such as France and Australia have prioritized hygiene promotion in their current WASH strategies. Agencies, such as BRAC and DFID, also mentioned working through various integrative programmes to enhance hygiene promotion as part of WASH activities.

\section{Discussion}

The results of the GLAAS survey showed that countries faced challenges in implementing polices and plans, and establishing and making use of targets, performance indicators and baseline data, to support governance and monitoring of these plans. Access to, and use of, financial plans and budgets to drive hygiene promotion was limited.

A critical aspect to global monitoring of hygiene promotion is how it is defined. The results varied greatly for how countries define hygiene promotion (Figure 2). We need a more detailed and robust analysis of how countries define hygiene promotion, to understand where current investments and priorities are directed, and to determine how to make better, evidence-based, contextappropriate decisions. For example, research provides evidence that hygiene promotion interventions reduce the risk of diarrhoea by $33 \%$ (RR $0.67,95 \%$ CI $0.61-0.74$ ). When stratified by specific activity, hand washing with soap resulted in a reduction of diarrhoea by $40 \%$ (RR $0.60,95 \%$ CI $0.53-0.68)$ and hygiene education alone by $24 \%$ (RR 0.76, 95\% CI 0.67-0.86) [3]. There are additional hygiene promotion factors that are likely to have an impact on improved health outcomes, for example safe food hygiene and menstrual hygiene management $[14,15]$.

The results also point to a gap between the development and implementation of national hygiene promotion plans and policies, with only $21 \%$ of countries implementing existing policies. While the data provided do not allow for the researchers to determine what key factors contributed to these bottlenecks, survey results do indicate a lack of coordination mechanisms between ministries, NGOs, the private sector, academia and other development actors working in hygiene promotion. 
Others have also cited a lack of political will to champion hygiene promotion as a critical factor [16]. With various international actions to improve WASH sector coordination, through initiatives such as the Global Framework for Action on Water and Sanitation, and the Sanitation and Water for All (SWA) Partnership, there are mechanisms in place to assist with improvements in sector coordination [17]. Again, more detailed analysis at a country level of what these limiting factors are in successful execution of hygiene promotion plans and policies is warranted.

Weak participation by user groups in the planning of hygiene promotion programmes also limits the potential to sustain gains [18]. Sustained behaviour improvements require intensive engagement of different community groups and high levels of practice in the community [18]. This aligns with findings from the GLAAS survey that community mobilisation is an area that would benefit most from additional human resources [19]. This question had one of the highest response rates for all hygiene-specific questions, yet no details were requested, and thus not provided, to confirm in what policy or law these procedures exist. The question also did not ask for respondents to stratify by type of group for participants, failing to provide useful information to determine if policies are targeting the most disadvantaged groups. This is important given the inequality that often exists in participation in, and control over, WASH-based decisions, and in particular, the gender inequalities in responsibility for hygiene promotion by women [20].

The lack of hygiene promotion budgets, or lack of adherence thereto, impede governments' ability to track actual $v s$. committed expenditures, to monitor cost-effectiveness of programme interventions, and to make appropriate allocations in future budgets. This contributes to the continuous underfunding and neglect of hygiene promotion in the WASH and greater public health context. This is a challenge given that it may not always be clear under what budget line the cost falls. For example, infrastructure may go under a water and/or sanitation budget line, but investment in these two areas also has an impact on hygiene. The UN-Water GLAAS TrackFin Initiative, an effort resulting from previous GLAAS results indicating that here are substantial gaps in our understanding and tracking of financing to the WASH sector, confirms that if hygiene financing is to be adequately tracked, specific international classifications need to be developed [12]. It also suggests that the challenge in tracking hygiene promotion expenditures stems from a lack of being able to define these [12]. Given that so few countries provided budgets, the $66 \%$ that state that they have finance plans and/or budgets for hygiene promotion should be questioned.

Supporting the lack of implementation of policies and plans was the low overall priority placed on hygiene promotion by the international external support/donor community. Underlying the findings mentioned above is the lack of information available to analyse and identify the specific bottlenecks that limit progress in the areas of governance, monitoring and finance. Further in-depth research is needed to ascertain information about what factors contribute to these bottlenecks and sound methodological approaches must be employed to gather these data. Only then will a better picture emerge about what variables contribute to improving hygiene promotion inputs and how these inputs can be harnessed to improve hygiene promotion outputs to improve people's health.

While the GLAAS survey is one of the few tools available used to collect national-level data for hygiene promotion, due to various data limitations, care should be taken when interpreting results. There were missing data for almost every question, with an average response rate of $52 \%$. Therefore, there was limited scope to conduct in-depth analysis between countries or regions due to the wide variance in the number of countries responding to each question (ranged from $4 \%$ to $100 \%$ ). Results may have been affected by reporting bias, data incompleteness and limited validation; thus, care must be taken when interpreting these findings.

\section{Conclusion}

Further research is needed to better understand what key activities are part of hygiene promotion, stratified by household, school and healthcare facility, and why countries struggle to fully implement policies and plans. Collection of data for institutional-level hygiene promotion inputs is of particular importance given the proposed targets in the SDGs that focus on hand washing with soap at both schools and healthcare facilities [13]. Additional research also needs to be conducted to understand government priorities for hygiene promotion alongside existing activities conducted by government, NGOs and the private sector. This will provide a framework to help determine the bottlenecks contributing to the lack of implementation by governments of hygiene promotion plans and policies in relation to findings from the GLAAS survey. Donors and governments should consider investing in additional human resources to target community mobilisation specifically through hygiene promotion initiatives. More research is required in order to determine 
where best to target this investment, giving consideration to equity and the differing hygiene promotion needs in rural and in urban environments. Finally, the GLAAS survey could be improved to assist with global monitoring and building evidence about the status of extending and sustaining hygiene promotion outcomes through the WASH sector by:

- Information more methodically about the ways in which countries define hygiene promotion, and link this to the questions about the existence of policies, targets and indicators;

- Gathering data to assess whether there is sufficient financing to meet country-established targets for hygiene promotion, and if not, ask countries to indicate in what specific areas additional funding is required and the amount that is necessary to meet targets. Link this with existing results from questions related to constraints to WASH HR and to proposed targets set for the SDGs;

- Cross-referencing output results with key inputs to assess the influence of these inputs on performance cross-reference output results with key inputs to assess the influence of these inputs on performance, as more data becomes available through the Joint Monitoring Programme (JMP) for Water Supply and Sanitation for the post-2015 era and through the DHS and MICS related to health promotion;

- Encouraging, through support of the GLAAS focal person and WHO country regional offices, the validation of country-level data from that region in order to enhance the quality and validity of hygiene promotion data used in the report.

The results from the GLAAS 2013/2014 cycle confirm evidence that policy makers increasingly acknowledge the need of investing in hygiene promotion in addition to the hardware components of WASH interventions [21]. One of the main lessons learnt, reported by several countries participating in the GLAAS 2013/2014 survey, is how little they knew about what was happening in relation to hygiene and have taken this on board as an important WASH action. There is already evidence that hygiene promotion is gaining more attention. For example, at the 2014 SWA meeting, 43 countries made a total of 309 commitments [22]. Several of these countries specifically committed to at least one hygiene-specific action. Monitoring these commitments through data collected as part of the GLAAS process can help build evidence about the drivers enabling, or disabling, countries in achieving set targets, goals and commitments for hygiene promotion, bringing further attention to this seemingly neglected issue. Given the important role hygiene promotion plays in improving health and saving lives, understanding the evidence base and the trends is a critical part of advancing the public health agenda.

\section{Acknowledgements}

We would like to thank the GLAAS team, including Bruce Gordon (Water, Sanitation, Hygiene and Health, Coordinator, WHO), Didier Allély, Betsey Engebretson, Mark Hoeke, Cathy Jung, Tara Neville and Peregrine Swann, for the coordination and development of the GLAAS 2014 Report. Thanks to all of the individuals, partners and organisations that contributed to the development of the results. The authors alone are responsible for the views expressed in this article, and they do not necessarily represent the views, decisions or policies of the institutions with which they are affiliated. This article is part of a multiple piece sequence that expands upon quantitative and/or qualitative data from the World Health Organization (WHO)-led initiative UN-Water Global Analysis and Assessment of Sanitation and Drinking-Water (GLAAS)' cycles from 2009/2010, 2011/2012 and 2013/2014. GLAAS data focus on the enabling environment (laws, plans and policies, institutional arrangements and monitoring systems) for water, sanitation and hygiene (WASH). While the biennial GLAAS report provides a broader overview of the enabling environment, these articles use the same data to generate perspectives that provide deeper analysis and insight into specific WASH topics that can be used for advocacy efforts as well as for strengthening the WASH sector both locally and globally. For more information, please contact glaas@who.int

\section{References}

1. Laxminarayan R, Chow J, Shahid-Salles SA. Disease Control Priorities in Developing Countries (2nd edn), Oxford University Press: New York, NY, 2006.

2. Prüss-Ustün A, Bartram J, Clasen T et al. Burden of disease from inadequate water, sanitation and hygiene in low- and middle-income settings: a retrospective analysis of data from 145 countries. Tropical Med Int Health 2014: 19: 894-905.

3. Freeman MC, Stocks ME, Cumming O et al. Systematic review: Hygiene and health: systematic review of handwashing practices worldwide and update of health effects. Tropical Med Int Health 2014: 19: 906-916.

4. Greenland K, Cairncross S, Cumming O, Curtis V. Editorial: can we afford to overlook hand hygiene again? Tropical Med Int Health 2013: 18: 246-249.

5. WHO. GLAAS 2010 report: Targeting resources for better results, 2010. 
L. D. Moreland et al. Monitoring sustained hygiene promotion

6. WHO. The Power Of Evidence: The UN-Water GLAAS Strategy 2010-2015. World Health Organization and UNWater: Geneva, Switzerland, 2010.

7. Jiménez A, Cavill S, Cairncross S. The neglect of hygiene promotion in developing countries, as shown by the Global Analysis and Assessment of Sanitation and Drinking-Water survey. J Water Sanit Hyg De 2014: 4: 240.

8. WHO. GLAAS 2008 report: 2008 Pilot Report - Testing a new reporting approach, 2008.

9. WHO. GLAAS 2012 report: The Challenge of Extending and Sustaining Services, World Health Organization, WHO, 2012.

10. WHO. Global Burden of Disease Facsheet: The top 10 causes of death [Online], 2014. (Available from: http:// www.who.int/mediacentre/factsheets/fs310/en/) [17 Aug 2014].

11. WHO. Investing in Water and Sanitation: Increasing Access, Reducing Inequalities. Special Report for the Sanitation and Water for All (SWA) High-Level Meeting (HLM) 2014 [Online], 2014. (Available from: http://www.who.int/water_sanitation_health/glaas/glaas_report_2014/en/) [15 April 2014].

12. WHO. UN-Water GLAAS TrackFin Initiative: Tracking financing to sanitation, hygiene and drinking-water at national level. WHO: Geneva, Switzerland, 2014.

13. WSSCC. WASH POST-2015: proposed targets and indicators for drinking-water, sanitation and hygiene [Online], 2014. (Available from: http://www.wsscc.org/resources/ resource-advocacy-materials/fact-sheets-post-2015-washtargets-and-indicators) [20 May 2014].

14. Biran A, Curtis V, Gautam OP et al. Background Paper on Measuring WASH and Food Hygiene Practices - Definition of Goals to be Tackled Post 2015 by the Joint Monitoring Programme. 2012.

15. Brown J, Cairncross S, Ensink JH. Water, sanitation, hygiene and enteric infections in children. Arch Dis Child 2013: 98: 629-634.

16. Cairncross S, Bartram J, Cumming O, Brocklehurst C. Hygiene, sanitation, and water: what needs to be done? PLoS Med 2010: 7: e1000365.

17. Dietvorst C, Cross P. International WASH cooperation past and future: an insider's view, 2013. (Available from: http://www.ircwash.org/news/international-wash-coopera tion- $\%$ E2 $\% 80 \% 93$-past-and-future-insider $\%$ E2\% $80 \% 99$ sview) [10 Aug 2014].

18. Shordt K. Sustaining Hygiene Behaviours [Online], 2003. (Available from: http://www.lboro.ac.uk/well/resources/factsheets/fact-sheets-htm/shb.htm) [08 Aug 2014].

19. Swann P, Souter R. Investing in water and sanitation: increasing access, reducing inequalities: Human Resources Chapter (proposed). World Health Organization and UNWater, 2014.

20. WaterAID and UWASNET. Mainstreaming gender in sanitation and hygiene in Uganda. Sanitation and Hygiene Conference. South Africa, 2002.

21. Cairncross S, Valdmanis V. Water supply, sanitation and hygiene promotion. In: Jamison DT, Breman JG, Measham AR, Alleyne G, Claeson M, Evans DB, Jha P, Mills A, Musgrove $\mathrm{P}$ (eds). Disease Control Priorities in Developing Countries, 2nd edn. World Bank Publications: Washington D.C., 2006.

22. SWA. Sanitation and Water for All [Online], 2014. (Available from: http://sanitationandwaterforall.org/commitments) [22 July 2014].

Corresponding Author Leslie D. Moreland, Faculty of Infectious and Tropical Diseases, London School of Hygiene and Tropical Medicine, Keppel Street, London WC1E 7HT, UK. Tel.: +832 213-9709; E-mail: ldmoreland@gmail.com 